\title{
EXPRESSING UNIPOTENT MATRICES OVER RINGS AS PRODUCTS OF INVOLUTIONS
}

\author{
Thomas J. Laffey
}

Let $R$ be a ring with 1 and let $M_{n}(R)$ be the ring of $n \times n$ matrices with entries in $R$. An element $A \in M_{n}(R)$ is called unipotent if it is of the form $I_{n}+N$ where $I_{n}$ is the identity $n \times n$ matrix and $N$ is either strictly upper triangular or strictly lower triangular. An element $J \in M_{n}(R)$ is called an involution if $J^{2}=I_{n}$. In this note we prove:

Theorem 1 Let $R$ be a ring and $n$ a positive integer. Then every unipotent element $A$ in $M_{n}(R)$ is the product of ten involutions. Proof: We can assume $A$ is upper triangular, say

$$
A=\left(\begin{array}{ccccc}
1 & a_{12} & a_{13} & \cdots & a_{1 n} \\
0 & 1 & a_{23} & \cdots & a_{2 n} \\
\vdots & \ddots & & & \\
0 & \cdots & 0 & 1 & a_{n-1 n} \\
0 & \cdots & 0 & 0 & 1
\end{array}\right)
$$

Write $A=H K$ where

$$
H=\left(\begin{array}{cccccccc}
1 & a_{12} & a_{12} a_{23} & 0 & \cdots & \cdots & \cdots & 0 \\
0 & 1 & a_{23} & 0 & \cdots & \cdots & \cdots & 0 \\
0 & 0 & 1 & a_{34} & a_{34} a_{45} & 0 & \cdots & 0 \\
0 & 0 & 0 & 1 & a_{45} & 0 & \cdots & 0 \\
\vdots & & & & \ddots & & & \\
\vdots & & & & & \ddots & & \\
0 & & & & & & 1 & a_{n-1 n} \\
0 & \cdots & \ldots & \ldots & \ldots & \cdots & 0 & 1
\end{array}\right)
$$


For $j$ odd, row $j$ of $H$ is of the form

$$
\left(0,0, \ldots, 0,1, a_{j j+1}, a_{j+1}{ }_{j+2}, \ldots, 0\right)
$$

while for $j$ even, it has the form

$$
\left(0,0, \ldots, 1, a_{j j+1}, 0, \ldots, 0\right) .
$$

Note that $K=H^{-1} A$ has the form

$$
K=\left(\begin{array}{cccccc}
1 & 0 & k_{13} & \cdots & \cdots & k_{1 n} \\
0 & 1 & 0 & k_{24} & \cdots & k_{2 n} \\
& & \ddots & & & \vdots \\
& & & \ddots & & k_{n-2 n} \\
& & & & \ddots & 0 \\
0 & 0 & & & 0 & 1
\end{array}\right)
$$

Note that $H$ is expressible as product $H=H_{1} H_{2}$, where

$$
H_{1}=\left(\begin{array}{cccccccc}
1 & a_{12} & 0 & \ldots & \ldots & \ldots & \cdots & 0 \\
0 & 1 & 0 & \ldots & \ldots & \ldots & \cdots & 0 \\
0 & 0 & 1 & a_{34} & 0 & \cdots & \cdots & 0 \\
0 & 0 & 0 & 1 & 0 & & \cdots & 0 \\
0 & 0 & 0 & 0 & 1 & a_{56} & \cdots & 0 \\
\vdots & & & & & \ddots & & \vdots \\
\vdots & & & & & & \ddots & \vdots \\
0 & \ldots & \ldots & \ldots & \ldots & \ldots & 0 & 1
\end{array}\right),
$$

and

$$
H_{2}=\left(\begin{array}{cccccccc}
1 & 0 & \ldots & \cdots & \cdots & \cdots & \cdots & 0 \\
0 & 1 & a_{34} & 0 & \cdots & \cdots & \cdots & 0 \\
0 & 0 & 1 & 0 & & \cdots & \cdots & 0 \\
0 & 0 & 0 & 1 & a_{45} & 0 & \cdots & 0 \\
0 & 0 & 0 & 0 & 1 & 0 & \cdots & 0 \\
\vdots & & & & & \ddots & & \vdots \\
\vdots & & & & & & \ddots & \vdots \\
0 & \cdots & \ldots & \ldots & \cdots & \cdots & 0 & 1
\end{array}\right)
$$


Using the factorization

$$
\left(\begin{array}{ll}
1 & \alpha \\
0 & 1
\end{array}\right)=\left(\begin{array}{rr}
1 & -\alpha \\
0 & -1
\end{array}\right)\left(\begin{array}{rr}
1 & 0 \\
0 & -1
\end{array}\right)
$$

where the two matrices on the right are involutions, we see that $H$ is the product of four involutions.

Note that

$$
K=J L
$$

where $J$ is the full Jordan block

$$
\left(\begin{array}{ccccccc}
1 & 1 & 0 & \cdots & \cdots & \cdots & 0 \\
0 & 1 & 1 & 0 & \cdots & \cdots & 0 \\
& & \ddots & \ddots & & & \vdots \\
& & & \ddots & \ddots & & \vdots \\
& & & & \ddots & \ddots & 0 \\
& & & & & 1 & 1 \\
0 & 0 & \cdots & \cdots & \cdots & 0 & 1
\end{array}\right)
$$

and $L$ is of the form

$$
\left(\begin{array}{ccccccc}
1 & -1 & l_{13} & \ldots & \cdots & \cdots & l_{1 n} \\
0 & 1 & -1 & l_{24} & \cdots & \cdots & l_{2 n} \\
& & \ddots & \ddots & & & \vdots \\
& & & \ddots & \ddots & & \vdots \\
& & & & \ddots & \ddots & 0 \\
& & & & & 1 & -1 \\
0 & 0 & \cdots & \cdots & \cdots & 0 & 1
\end{array}\right) .
$$

If $D$ is the diagonal matrix $\operatorname{diag}\left(1,-1,1,-1, \ldots,(-1)^{n-1}\right)$ then we define the matrix $L_{0}$ by

$$
L_{0}=D^{-1} L D=\left(\begin{array}{cccccc}
1 & 1 & l_{13} & -l_{14} & \cdots & \cdots \\
0 & 1 & 1 & l_{24} & \cdots & \vdots \\
\vdots & & \ddots & & & \vdots \\
\vdots & & & \ddots & & l_{n-2 n} \\
0 & & & 0 & 1 & 1 \\
0 & \cdots & \ldots & 0 & 0 & 1
\end{array}\right) .
$$


By a result of Dennis and Vaserstein, $L_{0}$ is similar to $J$ via an element $T$ of $G L(n, R)$. In fact, just take $T$ to be a unipotent upper-triangular matrix and observe that the linear system

$$
T L_{0}=J T
$$

is solvable inductively over every ring. (See [2], proof of Lemma 13.)

The desired result then follows from our next result.

Proposition The full unipotent Jordan block $J$ is the product of three involutions.

Proof: Let $C$ be the companion matrix of $(x-1)^{n}$, so

$$
C=\left(\begin{array}{ccccccc}
0 & 1 & 0 & \ldots & \ldots & \ldots & 0 \\
0 & 0 & 1 & 0 & \ldots & \ldots & 0 \\
& & & \ddots & & & \vdots \\
& & & & \ddots & & \vdots \\
& & & & & \ddots & \vdots \\
0 & \ldots & \ldots & \ldots & \ldots & \ldots & 1 \\
(-1)^{n-1} & \ldots & \ldots & \ldots & \left(\begin{array}{l}
n \\
3
\end{array}\right) & -\left(\begin{array}{c}
n \\
2
\end{array}\right) & n
\end{array}\right)
$$

Put

$$
\begin{aligned}
v_{1} & =(0,0, \ldots, 0,1)^{T} \\
v_{2} & =C v_{1}-v_{1}=(0,0, \ldots, 0,1, n-1)^{T} \\
v_{3} & =C v_{2}-v_{2}=\left(0,0, \ldots, 0,1, n-2,\left(\begin{array}{c}
n-1 \\
2
\end{array}\right)\right)^{T}, \\
v_{4} & =C v_{3}-v_{3}=\left(0,0, \ldots, 0,1, n-3,\left(\begin{array}{c}
n-2 \\
2
\end{array}\right),\left(\begin{array}{c}
n-1 \\
3
\end{array}\right)\right)^{T}, \\
\vdots & \quad \vdots \\
v_{n} & =C v_{n-1}-v_{n-1}=(1,1, \ldots, 1)^{T},
\end{aligned}
$$

and let $Q=\left(v_{1} v_{2} \cdots v_{n}\right)$. Then $\operatorname{det} Q=(-1)^{\left(\begin{array}{c}n \\ 2\end{array}\right)}$ and $C Q=Q J$. 
So $J$ is similar to $C$ over $R$.

But $C$ is expressible as the product $C=C_{1} C_{2}$, where

and

$$
C_{1}=\left(\begin{array}{ccccccc}
1 & 0 & \cdots & \cdots & \cdots & \cdots & 0 \\
0 & 1 & 0 & \cdots & \cdots & \cdots & 0 \\
\vdots & & \ddots & & & & \vdots \\
\vdots & & & \ddots & & & \vdots \\
\vdots & & & & \ddots & & \vdots \\
0 & \cdots & \cdots & \cdots & \cdots & 1 & 0 \\
& \cdots & \cdots & \left(\begin{array}{l}
n \\
3
\end{array}\right) & -\left(\begin{array}{c}
n \\
2
\end{array}\right) & n & -1
\end{array}\right)
$$

$$
C_{2}=\left(\begin{array}{ccccccc}
0 & 1 & 0 & \cdots & \cdots & \cdots & 0 \\
0 & 0 & 1 & 0 & \cdots & \cdots & 0 \\
0 & 0 & 0 & 1 & & & 0 \\
\vdots & & & & \ddots & & \vdots \\
\vdots & & & & & \ddots & \vdots \\
0 & \cdots & \cdots & \cdots & \cdots & \cdots & 1 \\
(-1)^{n} & 0 & \cdots & \cdots & \cdots & \cdots & 0
\end{array}\right) .
$$

Now $C_{1}$ is an involution and $C_{2}$ is the product of two involutions (when $n$ is even, this follows from the fact that every permutation is the product of two involutions and for $n$ odd, we use a slight modification of this argument). See [3] for this factorization of a companion matrix.

Hence $C$ and therefore $J$ is the product of three involutions.

A well-known result of Gustafson, Halmos and Radjavi, [3], states that if $F$ is a field and $A \in M_{n}(F)$ is such that $\operatorname{det} A=$ \pm 1 , then $A$ is the product of four involutions in $G L(n, F)$, but in general not the product of fewer than four. A number of results on matrices $A$ which are the product of three involutions is presented by Liu, [5].

Ishibashi [4] has obtained a version of the Gustafson-HalmosRadjavi result for integer matrices. He proves that if $n>2$ and $A \in G L(n, \mathbf{Z})$, then $A$ is the product of $3 n+9$ involutions in $G L(n, \mathbf{Z})$. 
It can be deduced from Bass, [1], that if $n \geq 3$ and $A \in$ $G L(n, \mathbf{Z})$, then

$$
A=U_{1} L_{1} U_{2} L_{2}\left(\begin{array}{cc}
S & 0 \\
0 & I_{n-2}
\end{array}\right)
$$

where $U_{1}, U_{2}$ are unipotent upper-triangular and $L_{1}, L_{2}$ are unipotent lower-triangular integer matrices and $S \in G L(2, \mathbf{Z})$ (see also [2], Lemma 9). Since

$$
U_{1} L_{1} U_{2} L_{2}=\left(U_{1} L_{1} U_{1}^{-1}\right)\left(U_{1} U_{2}\right) L_{2}
$$

and $U_{1} U_{2}$ is unipotent upper-triangular, we deduce from the theorem that $U_{1} L_{1} U_{2} L_{2}$ is the product of 30 involutions and applying Ishibashi's result in the case $n=3$, we deduce that $A$ is the product of 48 involutions. Using Bass's result, Dennis and Vaserstein, [2], show that for $n$ sufficiently large (one can check that $n \geq 82$ will do), every $A \in S L(n, \mathbf{Z})$ can be written as a product $U_{1} \bar{L}_{1} U_{2} L_{2} U_{3} L_{3}$ where $U_{1}, U_{2}, U_{3}$ are unipotent upper-triangular and $L_{1}, L_{2}, L_{3}$ unipotent lower-triangular integer matrices. Note that

$$
U_{1} L_{1} U_{2} L_{2} U_{3} L_{3}=\left(U_{1} L_{1} U_{1}^{-1}\right)\left(\left(U_{1} U_{2}\right) L_{2}\left(U_{1} U_{2}\right)^{-1}\right)\left(U_{1} U_{2} U_{3}\right) L_{3}
$$

Using this and the fact that if $\operatorname{det} A=-1$ and

$$
D:=\operatorname{diag}(-1,1,1, \ldots, 1)
$$

then $D$ is an involution and $A D \in S L(n, \mathbf{Z})$, Theorem 1 yields:

Theorem 2 Every $A \in G L(n, \mathbf{Z})(n \geq 3)$ can be written as a product of 48 (or fewer) involutions in $G L(n, \mathbf{Z})$. For $n \geq 82$, this number can be reduced to 41 .

Remark (i) It would be interesting to get best possible bounds. (ii) Ishibashi, [4], shows that for elements in $G L(2, \mathbf{Z})$, no such bound is possible.

\section{References}

[1] H. Bass, K-theory and stable algebra, Publ. Math. IHES. 22 (1964), $5-60$. 
[2] R. K. Dennis and L. N. Vaserstein, On a question of M. Newman on the number of commutators, J. Algebra 118 (1988), 150-161.

[3] W. H. Gustafson, P. R. Halmos and H. Radjavi, Products of involutions, Collection of articles dedicated to Olga Taussky Todd. Linear Algebra Appl. 13 (1976), 157-162.

[4] H. Ishibashi, Involutory expressions of elements in $G L_{n}(Z)$ and $S L_{n}(Z)$, Linear Algebra Appl. 219 (1995), 165-177.

[5] Kang-Man Liu, Decomposition of matrices into three involutions, Linear Algebra Appl. 111 (1988), 1-24.

Thomas J. Laffey

Department of Mathematics,

University College Dublin,

Belfield,

Dublin 4,

Ireland. 